\title{
Three-dimensional Observation of Cell-Cell and Cell-Matrix Interactions during Myofibrillogenesis of Embryonic Heart Tube.
}

Shiraishi I*, Takamatsu T**, Borg TK***, Hamaoka $\mathrm{K}^{*}$.

*Department of Pediatric Cardiology and Nephrology

Kyoto Prefectural University of Medicine Graduate School of Medical Science

Kawaramachi-Hirokoji, Kamigyo, Kyoto, Japan 602-8566. isao@koto.kpu-m.ac.jp

**Department of Cell and Developmental Biology and Anatomy, University of South Carolina, Columbia, SC 29208, USA.

***Department of Pathology and Cell Regulation

Kyoto Prefectural University of Medicine Graduate School of Medical Science

Kawaramachi-Hirokoji, Kamigyo, Kyoto, Japan 602-8566.

We performed 3D observation of myofibril formation, cell-cell and cell-matrix adhesions of whole-mounted chicken embryonic heart tube by means of confocal scanning microscopy (CSLM). We found that 1) from the earliest stages of myofibrillogenesis, myofibrils in neighboring myocytes interconnected with one another and constituted circumferential alignments at the bottom of the inner myocardial cell layer. 2) The arrangement of myofibrils developed in association with changing cell-cell and cell-matrix adhesions. 3) Inhibition of FAK, which is a main substrate of tyrosine phosphorylation at the bottom of the inner cell layer, results in disruption of the integrity of the looping heart tubes (i.e., ballooning). 4) After the initiation of myofibrillogenesis (E 9.5 and later), small G protein rho localized at the z-band of the developing myofibrils both in the outer and the inner myocardial layer cells. Inhibition of rho by Y27632 inhibited formation of the tubular heart and perturbed myofibrillogenesis. These results suggest that cell-cell and cell-matrix interaction is crucial for myofibril formation and organization, and looping of the heart tube. 


\section{$\underline{\text { References }}$}

[1] Shiraishi I, et al. Cell-cell and cell-matrix adhesion during formation and arrangement of developing cardiac myofibrils. Etiology and morphogenesis of congenital heart disease. Futura Publ., New York. 2000, p175-179.

[2] Shiraishi I, et al. Vinculin is an essential component for normal myofibrillar arrangement in fetal mouse cardiac myocytes. J Mol Cell Cardiol. 1997;29:2041-52.

[3] Shiraishi I, et al. Temporal and spatial patterns of phosphotyrosine immunolocalization during cardiac myofibrillogenesis of the chicken embryo. Anat Embryol. 1997;196:81-9.

[4] Shiraishi I, et al. Three-dimensional observation of F-actin and expression of N-cadherin and fibronectin during cardiac looping of the chick embryo using CSLM. In Developmental Mechanism of Heart Disease. Futura Publ., 1995, pp471-476.

[5] Price RL et al. Local and regional variations in myofibrillar patterns in looping rat hearts. Anat Rec. 1996;245:83-93.

[6] Shiraishi I, et al. Three-dimensional observation with a confocal scanning laser microscope of fibronectin immunolabeling during cardiac looping in the chick embryo. Anat Embryol. 1995;191:183-9.

[7] Shiraishi I, et al. 3-D observation of N-cadherin expression during cardiac myofibrillogenesis of the chick embryo using a confocal laser scanning microscope. Anat Embryol. 1993;187:115-20.

[8] Shiraishi I, et al. 3-D observation of actin filaments during cardiac myofibrinogenesis in chick embryo using a confocal laser scanning microscope. Anat Embryol. 1992;185:401-8. 